\title{
Fano hypersurfaces and Calabi-Yau supermanifolds
}

\author{
Richard S. Garavuso, Maximilian Kreuzer \\ Institut für Theoretische Physik, Technische Universität Wien \\ Wiedner Hauptstrasse 8-10/136, A-1040 Vienna, Austria \\ E-mail: garavuso@hep.itp.tuwien.ac.at, kreuzer@hep.itp.tuwien.ac.at
}

\author{
Alexander Noll \\ Institut für Theoretische Physik, Technische Universität Graz \\ Petersgasse 16/II, A-8010 Graz, Austria \\ E-mail: hava@sbox.tugraz.at
}

\begin{abstract}
In this paper, we study the geometrical interpretations associated with Sethi's proposed general correspondence between $\mathcal{N}=2$ Landau-Ginzburg orbifolds with integral $\hat{c}$ and $\mathcal{N}=2$ nonlinear sigma models. We focus on the supervarieties associated with $\hat{c}=3$ Gepner models. In the process, we test a conjecture regarding the superdimension of the singular locus of these supervarieties. The supervarieties are defined by a hypersurface $\widetilde{W}=0$ in a weighted superprojective space and have vanishing super-first Chern class. Here, $\widetilde{W}$ is the modified superpotential obtained by adding as necessary to the Gepner superpotential a boson mass term and/or fermion bilinears so that the superdimension of the supervariety is equal to $\hat{c}$. When Sethi's proposal calls for adding fermion bilinears, setting the bosonic part of $\widetilde{W}$ (denoted by $\widetilde{W}_{\text {bos }}$ ) equal to zero defines a Fano hypersurface embedded in a weighted projective space. In this case, if the Newton polytope of $\widetilde{W}_{b o s}$ admits a nef partition, then the Landau-Ginzburg orbifold can be given a geometrical interpretation as a nonlinear sigma model on a complete intersection Calabi-Yau manifold. The complete intersection Calabi-Yau manifold should be equivalent to the Calabi-Yau supermanifold prescribed by Sethi's proposal.
\end{abstract}

Keywords: Sigma Models, Conformal Field Models in String Theory, String Duality. 


\section{Contents}

1. Introduction 1

2. Gepner/NLSM correspondence 4

3. Fermionic weights and the singular locus constraint 6

4. Analysis 9

5. Conclusion 15

A. Supervariety hypersurface families 16

\section{Introduction}

Mirror symmetry [1] is a duality between string theories propagating on distinct but mirror target spaces. Consider a string theory compactified on a Calabi-Yau manifold $X$ which is related by mirror symmetry to a string theory compactified on a Calabi-Yau manifold $Y$. The mirror map relates the Hodge numbers of $X$ and $Y$ by $h_{X}^{p, q}=h_{Y}^{\mathcal{D}-p, q}$, where $\mathcal{D}$ is the complex dimension of $X$ and $Y$. Thus, the mirror map identifies the complex structure moduli space of $X$ with the Kähler moduli space of $Y$ and vice-versa.

A rigid Calabi-Yau manifold has no complex structure moduli. The mirror of such a manifold has no Kähler moduli and hence cannot be a Kähler manifold in the conventional sense. Thus, Calabi-Yau manifolds cannot be the most general geometrical framework for understanding mirror symmetry. The first progress towards generalizing this framework came when Schimmrigk [2] suggested that higherdimensional Fano varieties could provide mirrors for rigid Calabi-Yau manifolds. The name "generalized Calabi-Yau" was introduced by Candelas et al. [3] for these mirror manifolds. Later progress came when Sethi [4] proposed a general correspondence between $\mathcal{N}=2$ Landau-Ginzburg orbifolds with integral $\hat{c} \equiv c / 3$ (where $c$ is the central charge) and $\mathcal{N}=2$ nonlinear sigma models. Here, the target space of the nonlinear sigma model is either a Calabi-Yau manifold or a Calabi-Yau supermanifold. Using this proposal, Sethi argued that the mirror of a rigid Calabi-Yau manifold is a Calabi-Yau supermanifold and hence mirror symmetry should be viewed as a relation among Calabi-Yau manifolds and Calabi-Yau supermanifolds alike. The bodies of the 
supermanifolds are the Fano varieties mentioned above. Witten [5] described $\mathcal{N}=2$ nonlinear sigma models on Calabi-Yau manifolds and $\mathcal{N}=2$ Landau-Ginzburg orbifolds as being different phases of $\mathcal{N}=2$ gauged linear sigma models. Sethi's work and [6] have led others [7] to study $\mathcal{N}=2$ gauged linear sigma models which have a phase described by an $\mathcal{N}=2$ nonlinear sigma model on a Calabi-Yau supermanifold. The gauged linear sigma model framework allows the argument establishing a correspondence between the nonlinear sigma model and the Landau-Ginzburg orbifold to be made more robust.

A Calabi-Yau supermanifold $M$ obtained from Sethi's proposed correspondence would be realized by resolving the singularities of a supervariety $\mathcal{M}$ embedded in a weighted superprojective space

$$
\mathbf{W S P}^{(n \mid 2 m)} \equiv \mathbf{W S P}\left(n_{z_{1}}, \ldots, n_{z_{n+1}} \mid n_{\eta_{1}}, \ldots, n_{\eta_{2 m}}\right) .
$$

Here, $\mathcal{M}$ is defined by the zero locus of a transverse ${ }^{1}$, quasihomogeneous superpotential $\widetilde{W}=\widetilde{W}\left(z_{\mu} ; \eta_{\alpha}\right)$, where

$$
z_{\mu} \simeq \lambda^{n_{z_{\mu}}} z_{\mu}(\mu=1, \ldots, n+1), \quad \eta_{\alpha} \simeq \lambda^{n_{\eta_{\alpha}}} \eta_{\alpha}(\alpha=1, \ldots, 2 m)
$$

are homogeneous bosonic and fermionic coordinates of weights $n_{z_{\mu}}$ and $n_{\eta_{\alpha}}$, respectively. Since $\widetilde{W}$ is quasihomogeneous, it satisfies

$$
\widetilde{W}\left(\lambda^{n_{z \mu}} z_{\mu} ; \lambda^{n_{\eta_{\alpha}}} \eta_{\alpha}\right)=\lambda^{d} \widetilde{W}\left(z_{\mu} ; \eta_{\alpha}\right)
$$

where $d$ is the degree of quasihomogeneity. The superpotential $\widetilde{W}$ is obtained from the transverse, quasihomogeneous superpotential $W=W\left(\Phi_{a}\right)$ of an $\mathcal{N}=2$ LandauGinzburg model with integral $\hat{c}$ by truncating each chiral superfield $\Phi_{a}(a=1, \ldots, N)$ to its lowest bosonic component $\phi_{a}$, setting $\phi_{a}=z_{a}$, and then adding boson mass terms $z_{N+1}^{2}+\cdots+z_{n+1}^{2}$ and/or fermion bilinears $\eta_{1} \eta_{2}+\cdots+\eta_{2 m-1} \eta_{2 m}$ to $W$ so that

$$
\widetilde{\mathcal{D}} \equiv(n+1)-2 m-2=\hat{c} .
$$

Here, $\widetilde{\mathcal{D}}$ is the superdimension of the Calabi-Yau supermanifold. The condition (1.4) allows a change of variables with constant Jacobian to be made such that one of the new variables appears only linearly in the modified superpotential $\widetilde{W}$. The change of variables is not one-to-one, so the modified theory must be orbifolded by the diagonal subgroup of its phase symmetries. Integrating the linear new variable out of the path integral for the modified action as a Lagrange multiplier yields a super-delta function constraint which corresponds to $\mathcal{M}$ having vanishing super-first Chern class.

When $m \neq 0$, setting the bosonic part of $\widetilde{W}$ (denoted by $\widetilde{W}_{b o s}$ ) equal to zero defines a Fano hypersurface $\mathcal{F}$ embedded in a weighted projective space

$$
\mathbf{W P}^{n} \equiv \mathbf{W P}\left(n_{z_{1}}, \ldots, n_{z_{n+1}}\right)
$$

\footnotetext{
${ }^{1}$ Transverse $\widetilde{W}$ means that $\widetilde{W}=0$ and $d \widetilde{W}=0$ have no common solution except at the origin.
} 
In this case, if the Newton polytope $\Delta$ corresponding to $\widetilde{W}_{\text {bos }}$ admits a nef partition $\Delta=\Delta_{1}+\cdots+\Delta_{r}$, then the Landau-Ginzburg orbifold can be given a geometrical interpretation as a nonlinear sigma model on a complete intersection Calabi-Yau manifold defined by equations $f_{i}=0(i=1, \ldots, r)$ [8]. Here, $\Delta_{i}$ is the Newton polytope of $f_{i}$. The complete intersection Calabi-Yau manifold should be equivalent (in the sense of [6]) to the Calabi-Yau supermanifold prescribed by Sethi's proposed correspondence. When $m=0$, the constraint $\widetilde{W}=0$ defines a Calabi-Yau variety $\mathcal{X}$ embedded in $\mathbf{W P}^{n}$. Resolving $\mathcal{X}$ would yield a Calabi-Yau manifold $X$ of complex dimension

$$
\mathcal{D} \equiv(n+1)-2=\hat{c} .
$$

The complex dimension of the singular locus of $\mathcal{X}$ satisfies 9

$$
0 \leq \operatorname{dim}(\operatorname{Sing}(\mathcal{X})) \leq \mathcal{D}-2, \quad \operatorname{Sing}(\mathcal{X})=\mathcal{X} \cap \operatorname{Sing}\left(\mathbf{W P}^{n}\right)
$$

To obtain a Calabi-Yau supermanifold $M$ by resolving the singularities of $\mathcal{M}$, one might infer from the discussion in 4 that the superdimension of the singular locus of $\mathcal{M}$ must satisfy

$$
\operatorname{sdim}(\operatorname{Sing}(\mathcal{M})) \leq \widetilde{\mathcal{D}}-1, \quad \operatorname{Sing}(\mathcal{M})=\mathcal{M} \cap \operatorname{Sing}\left(\mathbf{W S P}^{(n \mid 2 m)}\right) .
$$

The result (1.7) and the equivalence discussed in [6] suggests the following stronger conjecture:

Conjecture 1.1 To obtain a Calabi-Yau supermanifold $M$ by resolving the singularities of $\mathcal{M}$, the superdimension of the singular locus of $\mathcal{M}$ must satisfy

$$
\operatorname{sdim}(\operatorname{Sing}(\mathcal{M})) \leq \widetilde{\mathcal{D}}-2, \quad \operatorname{Sing}(\mathcal{M})=\mathcal{M} \cap \operatorname{Sing}\left(\mathbf{W S P}^{(n \mid 2 m)}\right) .
$$

In this paper, we will test the above conjecture for $\widetilde{\mathcal{D}}=3$. This will be achieved by studying the geometrical interpretations prescribed by Sethi's proposed correspondence for Gepner models [10 with $\hat{c}=3$. Since $\widetilde{W}$ is quasihomogeneous of degree $d$, the weights of the fermions in each fermion bilinear $\eta_{2 k-1} \eta_{2 k}(k=1, \ldots, m)$ must satisfy

$$
n_{\eta_{2 k-1}}+n_{\eta_{2 k}}=d
$$

Requiring either the singular locus constraint (1.8) or Conjecture 1.1 to hold further restricts the fermionic weights. We have written a computer program which allows these restrictions to be implemented. In principle, one could determine the fermionic weights by requiring agreement between the Hodge diamond of the Landau-Ginzburg orbifold and the Hodge diamond of $\mathcal{M}$. The former Hodge diamond can be computed using the techniques of [11] whereas insight into the structure of the latter Hodge diamond can be obtained from the heuristic approach of [4] based on orbifold considerations [12]. We will compare the fermionic weights obtained in this way with those obtained from our computer program. 
This paper is organized as follows: In Section 2, we discuss the application of Sethi's proposed Landau-Ginzburg orbifold/nonlinear sigma model correspondence to Gepner models. In Section [3, we describe how the singular locus constraint (1.8) restricts the fermionic weights and work through an example. It is a trivial step to replace (1.8) with Conjecture 1.1 in our computer program. The analysis of Section 1 compares the fermionic weights obtained from our computer program with those obtained from the cohomological approach described in the previous paragraph. Several examples are included which highlight the similarities and differences. Concluding remarks are given in Section 5. Finally, in the Appendix, we tabulate the families of hypersurfaces associated with our supervarieties. Here, the fermionic weights are determined with our computer program by requiring (1.8) and (1.9) to be satisfied. For each hypersurface family, the Hodge numbers $h^{1,1}$ and $h^{2,1}$ and the Euler number of the associated Landau-Ginzburg orbifold are given. When the Newton polytope corresponding to $\widetilde{W}_{b o s}$ admits a nef partition, this is indicated. We also indicate when the Newton polytope of $\widetilde{W}_{\text {bos }}$ is nonreflexive Gorenstein.

\section{Gepner/NLSM correspondence}

The worldsheet action for an $\mathcal{N}=2$ Landau-Ginzburg model is [13]

$$
S=\int d^{2} \mathbf{z} d^{4} \theta K\left(\Phi_{a}, \bar{\Phi}_{a}\right)+\left(\int d^{2} \mathbf{z} d^{2} \theta W\left(\Phi_{a}\right)+\text { c.c. }\right),
$$

Here, the integral involving the Kähler potential $K$ is called the $D$-term, the integral involving the superpotential $W$ is called the $F$-term, and $\Phi_{a}(a=1, \ldots, N)$ are chiral superfields. The $D$-term contains only irrelevant operators whereas the $F$ term contains relevant operators. Thus, the superpotential defines a universality class under renormalization group flow. Requiring the superpotential to be transverse and quasihomogeneous is believed to ensure the existence of a unique, nontrivial IR fixed point which is conformally invariant. At this fixed point, the action (2.1) provides a Lagrangian description of an $\mathcal{N}=2$ minimal model [13, 14, 15] with

$$
\hat{c}=2 \sum_{a=1}^{N}\left(\frac{1}{2}-q_{\Phi_{a}}\right), \quad q_{\Phi_{a}} \equiv n_{\Phi_{a}} / d .
$$

The 10,839 transverse, quasihomogeneous Landau-Ginzburg superpotentials corresponding to $\mathcal{N}=2$ superconformal theories with $\hat{c}=3$ were classified in [16]. A subset of these correspond to Gepner models with $\hat{c}=3$. A Gepner model [10 is a string model constructed as an orbifold of a tensor product of $\mathcal{N}=2$ minimal models. The central charge of the $i$ th $\mathcal{N}=2$ minimal model in the tensor product is

$$
c_{i}=\frac{3 k_{i}}{k_{i}+2} \quad\left(k_{i}=1,2, \ldots\right)
$$


where $k_{i}$ is the level of the $\mathcal{N}=2$ superconformal algebra [17]. To obtain an anomalyfree compactification to $D$ spacetime dimensions ( $D<10$, even), the internal contribution to the central charge must be

$$
c=\sum_{i} c_{i}=\frac{3}{2}(10-D) .
$$

The work of [18, 19] associated Calabi-Yau manifolds to a large class of Gepner models. Sethi's proposal [4] prescribes a geometrical interpretation for all Gepner models. This prescription is as follows:

1. Start by associating superpotential terms [14

- $W_{i}=x_{i}^{k_{i}+2}$ to $A$-models of level $k_{i}$,

- $W_{i}=x_{i}^{\frac{k_{i}}{2}+1}+x_{i} y_{i}^{2}$ to $D$-models of level $k_{i}$ (even),

- $W_{i}=x_{i}^{3}+y_{i}^{4}, W_{i}=x_{i}^{3}+x_{i} y_{i}^{3}$, and $W_{i}=x_{i}^{3}+y_{i}^{5}$ to $E_{6^{-}}, E_{7^{-}}$, and $E_{8^{-}}$models of level $k_{i}=10,16,28$, respectively.

The tensor product of $r$ subtheories yields a transverse, quasihomogeneous Gepner superpotential

$$
W=\sum_{i=1}^{r} W_{i}=\sum_{i=1}^{r}\left(x_{i}^{l_{x_{i}}}+x_{i}^{\bar{l}_{x_{i}}} y_{i}^{l_{y_{i}}}\right) .
$$

For $A$-models, $y_{i}=0$ and $l_{x_{i}}=k_{i}+2$. For $D$-models, $l_{x_{i}}=k_{i} / 2+1, \bar{l}_{x_{i}}=1$, and $l_{y_{i}}=2$. For $E_{6^{-}}, E_{7^{-}}$, and $E_{8^{-}}$models, $l_{x_{i}}=3,3,3, \bar{l}_{x_{i}}=0,1,0$, and $l_{y_{i}}=4,3,5$, respectively. In all cases,

$$
n_{x_{i}} l_{x_{i}}=d
$$

Additionally, for $D$ - and E-models,

$$
n_{x_{i}} \bar{l}_{x_{i}}+n_{y_{i}} l_{y_{i}}=d
$$

The $x_{i}$ and nonzero $y_{i}(i=1, \ldots, r)$ are identified with the $z_{a}(a=1, \ldots, N)$ described in the Introduction according to the convention $z_{1}=x_{1}$,

$$
z_{2}= \begin{cases}y_{1} & \left(y_{1} \neq 0\right) \\ x_{2} & \left(y_{1}=0\right)\end{cases}
$$

and so on.

2. Add as necessary to $W$ a single ${ }^{2}$ boson mass term $z^{2}$ and/or fermion bilinears $\eta_{1} \eta_{2}+\cdots+\eta_{2 m-1} \eta_{2 m}$ so that

$$
(n+1)-2 m-2=\hat{c} .
$$

\footnotetext{
${ }^{2}$ There are three possibilities for an arbitrary Landau-Ginzburg model with integral $\hat{c}$ :
} 
We thus obtain the modified superpotential

$$
\widetilde{W}=\left\{\begin{array}{l}
W \quad(\hat{c}=N-2) \\
W+z^{2} \quad(\hat{c}>N-2) \\
W+\sum_{k=1}^{m} \eta_{2 k-1} \eta_{2 k} \quad\left(\hat{c}<N-2, \sum_{a=1}^{N} q_{z_{a}} \text { integral }\right) \\
W+z^{2}+\sum_{k=1}^{m} \eta_{2 k-1} \eta_{2 k} \quad\left(\hat{c}<N-2, \sum_{a=1}^{N} q_{z_{a}} \text { half-integral }\right) .
\end{array}\right.
$$

The added fields have no effect on the chiral ring or the conformal fixed point to which the theory flows. The condition (2.8) allows a change of variables with constant Jacobian to be made such that one of the new variables appears only linearly in the modified superpotential. The change of variables is not one-to-one, so the modified theory must orbifolded by the diagonal subgroup of its phase symmetries. When $m=0(m \neq 0)$, integrating the linear new variable out of the path integral for the modified action as a Lagrange multiplier yields a (super-)delta function constraint which corresponds to the bosonic variety $\mathcal{X}$ (supervariety $\mathcal{M}$ ) defined by $\widetilde{W}=0$ having vanishing (super-)first Chern class. The first Chern class of $\mathcal{X}$ vanishes when

$$
\sum_{\mu=1}^{n+1} n_{z_{\mu}}-d=0
$$

whereas the super-first Chern class of $\mathcal{M}$ vanishes when

$$
\sum_{\mu=1}^{n+1} n_{z_{\mu}}-\sum_{\alpha=1}^{2 m} n_{\eta_{\alpha}}-d=0
$$

\section{Fermionic weights and the singular locus constraint}

As described in the Introduction, we require the fermionic weights to be consistent with the singular locus constraint (1.8) and the quasihomogeneity constraint (1.9). The restriction placed on the fermionic weights by the latter constraint is obvious. Let us now explain what consistency with the former constraint means.

The supervariety $\mathcal{M}$ defined by the hypersurface $\widetilde{W}=0$ has a $\mathbf{Z}_{p}$ fixed point set under the weighted projective identification (1.2) if and only if the following conditions are both satisfied:

- For $\hat{c}=N-2$, no fields need to be added.

- For $\hat{c}>N-2$, boson mass terms $z_{N+1}^{2}+\cdots+z_{n+1}^{2}$ are required.

- For $\hat{c}<N-2$, the condition that $\hat{c}$ be integral implies that the sum of the charges $\sum_{a=1}^{N} q_{z_{a}}$ can be either integral or half-integral. The former requires adding fermion bilinears $\eta_{1} \eta_{2}+$ $\cdots+\eta_{2 m-1} \eta_{2 m}$ whereas the latter requires adding a single boson mass term $z_{n+1}^{2}$ and fermion bilinears $\eta_{1} \eta_{2}+\cdots+\eta_{2 m-1} \eta_{2 m}$.

For Gepner models, $\hat{c}$ is never greater than $N-2$ by more than 1 . Thus, it is never necessary to add more than one boson mass term to the Gepner superpotential. 
1. The index set $B_{p} \equiv\left\{\mu \mid \lambda_{p}^{n_{z_{\mu}}}=1\right\}$ is nonempty, where $\lambda_{p} \equiv e^{2 \pi i / p}$.

2. The quantity

$$
\mathcal{D}_{p}= \begin{cases}\left|B_{p}\right|-2 & (d / p \in \mathbf{Z}) \\ \left|B_{p}\right|-1 & (d / p \notin \mathbf{Z}) .\end{cases}
$$

satisfies $\mathcal{D}_{p} \geq 0$ if the index set $F_{p} \equiv\left\{\alpha \mid \lambda_{p}^{n_{\eta_{\alpha}}}=1\right\}$ is empty.

There are no purely fermionic $\mathbf{Z}_{p}$ fixed point sets because the body of $\mathcal{M}$ is embedded in $\mathbf{W} \mathbf{P}^{n}$. The superdimension of the $\mathbf{Z}_{p}$ fixed point sets which do exist is given by

$$
\widetilde{\mathcal{D}}_{p}= \begin{cases}\left|B_{p}\right|-\left|F_{p}\right|-2 & (d / p \in \mathbf{Z}) \\ \left|B_{p}\right|-\left|F_{p}\right|-1 & (d / p \notin \mathbf{Z}) .\end{cases}
$$

In the case $d / p \in \mathbf{Z}$, the -2 arises because the weighted projective identification and the hypersurface equation each reduce the superdimension by 1 . In the case $d / p \notin \mathbf{Z}$, the hypersurface equation is identically satisfied and hence does not reduce the superdimension. Consistency with the singular locus constraint (1.8) means that

$$
\widetilde{\mathcal{D}}_{p} \leq \hat{c}-1 \quad \forall p
$$

where we have used (1.4). We see from (3.2) that $\mathbf{Z}_{p}$ fixed point sets with $\left|B_{p}\right| \leq$ $\left|F_{p}\right|$ have negative superdimension and hence are consistent with (1.8). Thus, when checking for consistency with (1.8), we can focus our attention on $\mathbf{Z}_{p}$ fixed point sets with $\left|B_{p}\right|>\left|F_{p}\right|$. Note that the bosonic part of such a $\mathbf{Z}_{p}$ fixed point set has complex dimension given by (3.1).

To illustrate the above, let us consider a concrete example.

Example 3.1 Consider a Gepner model with level/invariant structure $10_{D} 10_{D} 1_{A}$ $1_{A} 1_{A} 1_{A}$. Following the procedure described in Section Q, we obtain the quasihomogeneous degree $d=12$ modified superpotential

$$
\widetilde{W}=\sum_{i=1}^{2}\left(x_{i}^{6}+x_{i} y_{i}^{2}\right)+\sum_{i=3}^{6} x_{i}^{3}+z^{2}+\eta_{1} \eta_{2}+\eta_{3} \eta_{4} .
$$

The hypersurface $\widetilde{W}=0$ defines a supervariety $\mathcal{M}$ embedded in

$$
\mathbf{W S P}\left(2,5,2,5,4,4,4,4,6 \mid n_{\eta_{1}}, n_{\eta_{2}}, n_{\eta_{3}}, n_{\eta_{4}}\right) \text {. }
$$

We will denote the family of quasihomogeneous degree $d=12$ hypersurfaces embedded in this weighted superprojective space by

$$
\mathbf{W S P}\left(2,5,2,5,4,4,4,4,6 \mid n_{\eta_{1}}, n_{\eta_{2}}, n_{\eta_{3}}, n_{\eta_{4}}\right)[12] .
$$

A hypersurface in this family would also be obtained from a Gepner model with level and invariant structure $4_{D} 4_{D} 10_{D} 10_{D}$ or $4_{D} 10_{D} 10_{D} 1_{A} 1_{A}$. 
According to (1.9), the fermions in each bilinear can take on the values (modulo a relabelling of the fermions)

$$
\left(n_{\eta_{2 k-1}}, n_{\eta_{2 k}}\right) \in\{(1,11),(2,10),(3,9),(4,8),(5,7),(6,6)\} .
$$

To further constrain the fermionic weights, we now consider the $\mathbf{Z}_{p}(p=2,4,5)$ fixed point sets. First, we determine the complex dimension of the bosonic parts of these fixed point sets:

$\underline{p=2}$ : The bosonic part of the $\mathbf{Z}_{2}$ fixed point set is

$$
\sum_{i=1}^{2}\left(x_{i}^{6}+x_{i} y_{i}^{2}\right)+\sum_{i=3}^{6} x_{i}^{3}+z^{2}=0, \quad y_{1}=y_{2}=0 .
$$

There are $\left|B_{2}\right|=7$ bosons $\left(x_{i}(i=1, \ldots, 6)\right.$ and $\left.z\right)$ in this fixed point set. Since $d / p=6 \in \mathbf{Z}$, (3.1) gives

$$
\mathcal{D}_{2}=\left|B_{2}\right|-2=5
$$

$\underline{p=4}$ : The bosonic part of the $\mathbf{Z}_{4}$ fixed point set is

$$
\sum_{i=3}^{6} x_{i}^{3}=0, \quad x_{i}=y_{i}=0 \quad(i=1,2), \quad z=0 .
$$

There are $\left|B_{4}\right|=4$ bosons $\left(x_{i}(i=3, \ldots, 6)\right)$ in this fixed point set. Since $d / p=3 \in \mathbf{Z}$, 3.1 gives

$$
\mathcal{D}_{4}=\left|B_{4}\right|-2=2 \text {. }
$$

$\underline{p=5}$ : The bosonic part of the $\mathbf{Z}_{5}$ fixed point set is

$$
\sum_{i=1}^{2}\left(x_{i}^{6}+x_{i} y_{i}^{2}\right)=0, \quad x_{i}=0 \quad(i=1, \ldots, 6), \quad z=0 .
$$

There are $\left|B_{5}\right|=2$ bosons $\left(y_{1}\right.$ and $\left.y_{2}\right)$ in this fixed point set. Since $d / p=$ $12 / 5 \notin \mathbf{Z}$, 3.1) gives

$$
\mathcal{D}_{5}=\left|B_{5}\right|-1=1
$$

Next, let $\widetilde{\mathcal{D}}_{p}^{(j)}=\mathcal{D}_{p}-\left|F_{p}^{(j)}\right|$ be the superdimension of the $\mathbf{Z}_{p}$ fixed point set for the jth distinct (modulo relabelling) fermionic weight combination $\left(n_{\eta_{1}}, n_{\eta_{2}}, n_{\eta_{3}}, n_{\eta_{4}}\right)_{j}$ consistent with (1.9). For $m=2$ fermion bilinears, there are $\frac{1}{2}\left[\frac{d}{2}\right]\left(\left[\frac{d}{2}\right]+1\right)$ such combinations, where $[x]$ denotes the integer part of $x$. Thus, for this example, $j=$ $1, \ldots, 21$. The $\widetilde{\mathcal{D}}_{p}^{(j)}$ are given in Table 1 . Finally, exclude all fermionic weight combinations which do not satisfy

$$
\widetilde{\mathcal{D}}_{p}^{(j)} \leq \hat{c}-1=2 \forall p
$$




\begin{tabular}{rcccc}
$j$ & $\left(n_{\eta_{1}}, n_{\eta_{2}}, n_{\eta_{3}}, n_{\eta_{4}}\right)_{j}$ & $\widetilde{\mathcal{D}}_{2}^{(j)}$ & $\widetilde{\mathcal{D}}_{4}^{(j)}$ & $\widetilde{\mathcal{D}}_{5}^{(j)}$ \\
\hline 1 & $(1,11,1,11)$ & 5 & 2 & 1 \\
2 & $(1,11,2,10)$ & 3 & 2 & 0 \\
3 & $(1,11,3,9)$ & 5 & 2 & 1 \\
4 & $(1,11,4,8)$ & 3 & 0 & 1 \\
5 & $(1,11,5,7)$ & 5 & 2 & 0 \\
6 & $(1,11,6,6)$ & 3 & 2 & 1 \\
7 & $(2,10,2,10)$ & 1 & 2 & -1 \\
8 & $(2,10,3,9)$ & 3 & 2 & 0 \\
9 & $(2,10,4,8)$ & 1 & 0 & 0 \\
10 & $(2,10,5,7)$ & 3 & 2 & -1 \\
11 & $(2,10,6,6)$ & 1 & 2 & 0 \\
12 & $(3,9,3,9)$ & 5 & 2 & 1 \\
13 & $(3,9,4,8)$ & 3 & 0 & 1 \\
14 & $(3,9,5,7)$ & 5 & 2 & 0 \\
15 & $(3,9,6,6)$ & 3 & 2 & 1 \\
16 & $(4,8,4,8)$ & 1 & -2 & 1 \\
17 & $(4,8,5,7)$ & 3 & 0 & 0 \\
18 & $(4,8,6,6)$ & 1 & 0 & 1 \\
19 & $(5,7,5,7)$ & 5 & 2 & -1 \\
20 & $(5,7,6,6)$ & 3 & 2 & 0 \\
21 & $(6,6,6,6)$ & 1 & 2 & 1
\end{tabular}

Table 1: Computation of $\widetilde{\mathcal{D}}_{p}^{(j)}=\mathcal{D}_{p}-\left|F_{p}^{(j)}\right|(p=2,4,5)$.

This leaves the fermionic weight combinations

$$
(2,10,2,10),(2,10,4,8),(2,10,6,6),(4,8,4,8),(4,8,6,6),(6,6,6,6) .
$$

We can use the parameters $k$ and $l$ defined in the Appendix to express these combinations in the compact form $(2 k, 12-2 k, 2 l, 12-2 l)$. Here, $k=1, \ldots, 3$ and $l=1, \ldots, 3$. It is understood that repeated fermionic weight combinations generated with this notation are counted only once. In this notation, the hypersurface $\widetilde{W}=0$ defines a supervariety $\mathcal{M}$ embedded in $\mathbf{W S P}(2,5,2,5,4,4,4,4,6 \mid 2 k, 12-2 k, 2 l, 12-2 l)$ and is a member of hypersurface family 250 of the Appendix:

$$
\mathbf{W S P}(2,5,2,5,4,4,4,4,6 \mid 2 k, 12-2 k, 2 l, 12-2 l)[12] .
$$

\section{Analysis}

We have written a computer program which takes as input data which encodes the superpotential $W$ for $\hat{c}=3$ Gepner models. Next, the program determines the 
modified superpotential $\widetilde{W}$ as explained in Section 2. When $\widetilde{W}$ depends on five bosonic fields and no fermionic fields, the output of the program is the hypersurface family

$$
\mathbf{W P}\left(n_{z_{1}}, \ldots, n_{z_{n+1}}\right)[d]
$$

corresponding to the hypersurface $\widetilde{W}=0$ which defines a bosonic variety $\mathcal{X}$ embedded in $\mathbf{W P}\left(n_{z_{1}}, \ldots, n_{z_{n+1}}\right)$. For the remaining cases, the output is the hypersurface family

$$
\operatorname{WSP}\left(n_{z_{1}}, \ldots, n_{z_{n+1}} \mid n_{\eta_{1}}, \ldots, n_{\eta_{2 m}}\right)[d]
$$

corresponding to the hypersurface $\widetilde{W}=0$ which defines a supervariety $\mathcal{M}$ embedded in $\mathbf{W S P}\left(n_{z_{1}}, \ldots, n_{z_{n+1}} \mid n_{\eta_{1}}, \ldots, n_{\eta_{2 m}}\right)$. The fermionic weights are determined by requiring the singular locus constraint (1.8) and the quasihomogeneity constraint (1.9) to be satisfied. In this manner, we obtain at least one fermionic weight solution for each $\hat{c}=3$ Gepner model corresponding to a supervariety $\mathcal{M}$ through Sethi's proposed correspondence. The 254 hypersurface families associated with these supervarieties are tabulated in the Appendix. When we replace the singular locus constraint (1.8) with Conjecture 1.1, this results in models corresponding to the hypersurface families 50, 94, 95, 121, 125, and 229 of the Appendix having no solution for the fermionic weights.

In principle, the singularities which do arise could be determined by identifying the fermionic weights which yield agreement between the Hodge diamond of the Landau-Ginzburg orbifold and the Hodge diamond of $\mathcal{M}$. The Landau-Ginzburg orbifold Hodge diamond can be computed using the techniques of 11]. Such calculations can be done quickly with the software package PALP [20]. Unfortunately, at present, there is no supercohomology theory which allows the Hodge diamond of $\mathcal{M}$ to be computed.

The Hodge diamond of $\mathcal{X}$ can be computed using the orbifold techniques of [12]. This is possible because, with a change of coordinates

$$
z_{\mu}=\left(\zeta_{\mu}\right)^{n_{z_{\mu}}}
$$

the hypersurface $\mathbf{W P}\left(n_{z_{1}}, \ldots, n_{z_{n+1}}\right)[d]$ which defines $\mathcal{X}$ can be written as an orbifold of a hypersurface in a homogeneous projective space $\mathbf{P}^{n}$, i.e.

$$
\mathbf{W P}\left(n_{z_{1}}, \ldots, n_{z_{n+1}}\right)[d]=\frac{\mathbf{P}^{n}[d]}{\mathbf{Z}_{n_{z_{1}}} \times \cdots \times \mathbf{Z}_{n_{z_{n+1}}}} .
$$

In contrast, the hypersurface $\mathbf{W S P}\left(n_{z_{1}}, \ldots, n_{z_{n+1}} \mid n_{\eta_{1}}, \ldots, n_{\eta_{2 m}}\right)[d]$ which defines $\mathcal{M}$ cannot be written as an orbifold of a hypersurface in homogeneous superprojective space $\mathbf{S P}^{(n \mid 2 m)}$. This is because the analogue of (4.1) does not make sense for Grassmann coordinates. Nevertheless, as described in [4], we can use orbifold considerations to gain insight into the structure of the Hodge diamond of $\mathcal{M}$. In the 
following examples, we will use this heuristic reasoning and compare the resulting fermionic weights with those obtained from the singular locus constraint (1.8) and Conjecture 1.1.

Example 4.1 A hypersurface in the family $\mathbf{W S P}\left(1,1,2,2,2,2,2 \mid n_{\eta_{1}}, n_{\eta_{2}}\right)[6]$ can be obtained from a Gepner model with any of the following level/invariant structures:

$$
4_{D} 4_{D} 4_{A} 4_{A} 1_{A}, \quad 4_{D} 4_{A} 4_{A} 1_{A} 1_{A} 1_{A}, \quad 4_{A} 4_{A} 1_{A} 1_{A} 1_{A} 1_{A} 1_{A}
$$

For definiteness, we will focus on the last of these. Following the procedure described in Section 2 , we obtain the modified superpotential

$$
\widetilde{W}=x_{1}^{6}+x_{2}^{6}+x_{3}^{3}+x_{4}^{3}+x_{5}^{3}+x_{6}^{3}+x_{7}^{3}+\eta_{1} \eta_{2} \text {. }
$$

This example was discussed in [4]. Here, we simply note that cohomology considerations, Conjecture 1.1, and, as indicated by hypersurface family 179 of the Appendix, the singular locus constraint (1.8) all yield the same unique result $\left(n_{\eta_{1}}, n_{\eta_{2}}\right)=(2,4)$.

Example 4.2 A hypersurface in the family $\mathbf{W S P}\left(1,1,4,4,4,4,6 \mid n_{\eta_{1}}, n_{\eta_{2}}\right)[12]$ can be obtained from a Gepner model with any of the following level/invariant structures:

$$
\begin{aligned}
& 4_{D} 10_{A} 4_{A} 2_{A} 1_{A}, \quad 10_{A} 4_{A} 2_{A} 1_{A} 1_{A} 1_{A}, \quad 4_{D} 4_{D} 10_{A} 10_{A} \text {, } \\
& \begin{array}{lllllllllll}
4_{D} & 10_{A} & 10_{A} & 1_{A} & 1_{A}, & 10_{A} & 10_{A} & 1_{A} & 1_{A} & 1_{A} & 1_{A}
\end{array} .
\end{aligned}
$$

For definiteness, we will focus on the last of these. Following the procedure described in Section 2, we obtain the modified superpotential

$$
\widetilde{W}=x_{1}^{12}+x_{2}^{12}+x_{3}^{3}+x_{4}^{3}+x_{5}^{3}+x_{6}^{3}+z^{2}+\eta_{1} \eta_{2} \text {. }
$$

Employing the heuristic reasoning of [4], we find for $\left(n_{\eta_{1}}, n_{\eta_{2}}\right)=(4,8)$ that the Hodge diamond of $\mathcal{M}$ is

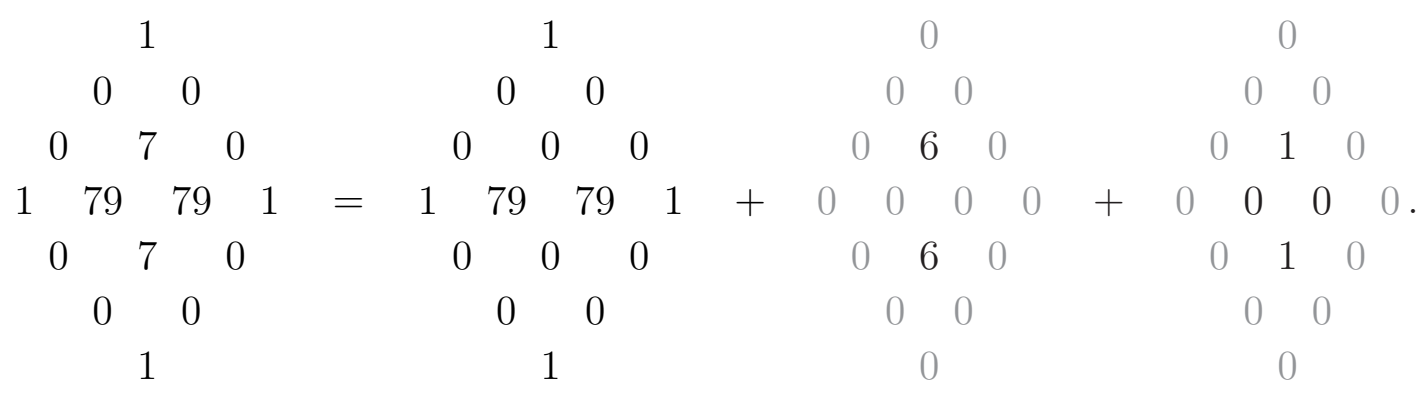

The first term on the right-hand side of (4.3) is the contribution arising from the untwisted sector. The second term includes the contribution of the fixed point set associated with the third twisted sector (the upper 6) and the fixed point set associated 
with the ninth twisted sector (the lower 6). Finally, the last term arises from the identity and volume forms of the fixed point set associated with the sixth twisted sector. Our result (4.3) agrees with the Hodge diamond of the associated LandauGinzburg orbifold. For all other fermionic weight assigments consistent with (1.9), we do not obtain this agreement. Thus, in this example, using these heuristic arguments, we obtain a unique result

$$
\left(n_{\eta_{1}}, n_{\eta_{2}}\right)=(4,8) \text {. }
$$

We note that this result agrees with what would be obtained from Conjecture 1.1. In contrast, as indicated by hypersurface family 10 of the Appendix, the singular locus constraint (1.8) allows

$$
\left(n_{\eta_{1}}, n_{\eta_{2}}\right) \in\{(2,10),(4,8),(6,6)\}
$$

Example 4.3 A hypersurface in the family $\mathbf{W S P}\left(1,3,3,3,4,4,6 \mid n_{\eta_{1}}, n_{\eta_{2}}\right)[12]$ can be obtained from a Gepner model with either of the following level/invariant structures:

$$
4_{D} 10_{A} 2_{A} 2_{A} 2_{A}, \quad 10_{A} 2_{A} 2_{A} 2_{A} 1_{A} 1_{A} .
$$

For definiteness, we will focus on the last of these. Following the procedure described in Section 2, we obtain the modified superpotential

$$
\widetilde{W}=x_{1}^{12}+x_{2}^{4}+x_{3}^{4}+x_{4}^{4}+x_{5}^{3}+x_{6}^{3}+z^{2}+\eta_{1} \eta_{2} \text {. }
$$

Employing the heuristic reasoning of [4], we find for $\left(n_{\eta_{1}}, n_{\eta_{2}}\right)=(3,9)$ that the Hodge diamond of $\mathcal{M}$ is

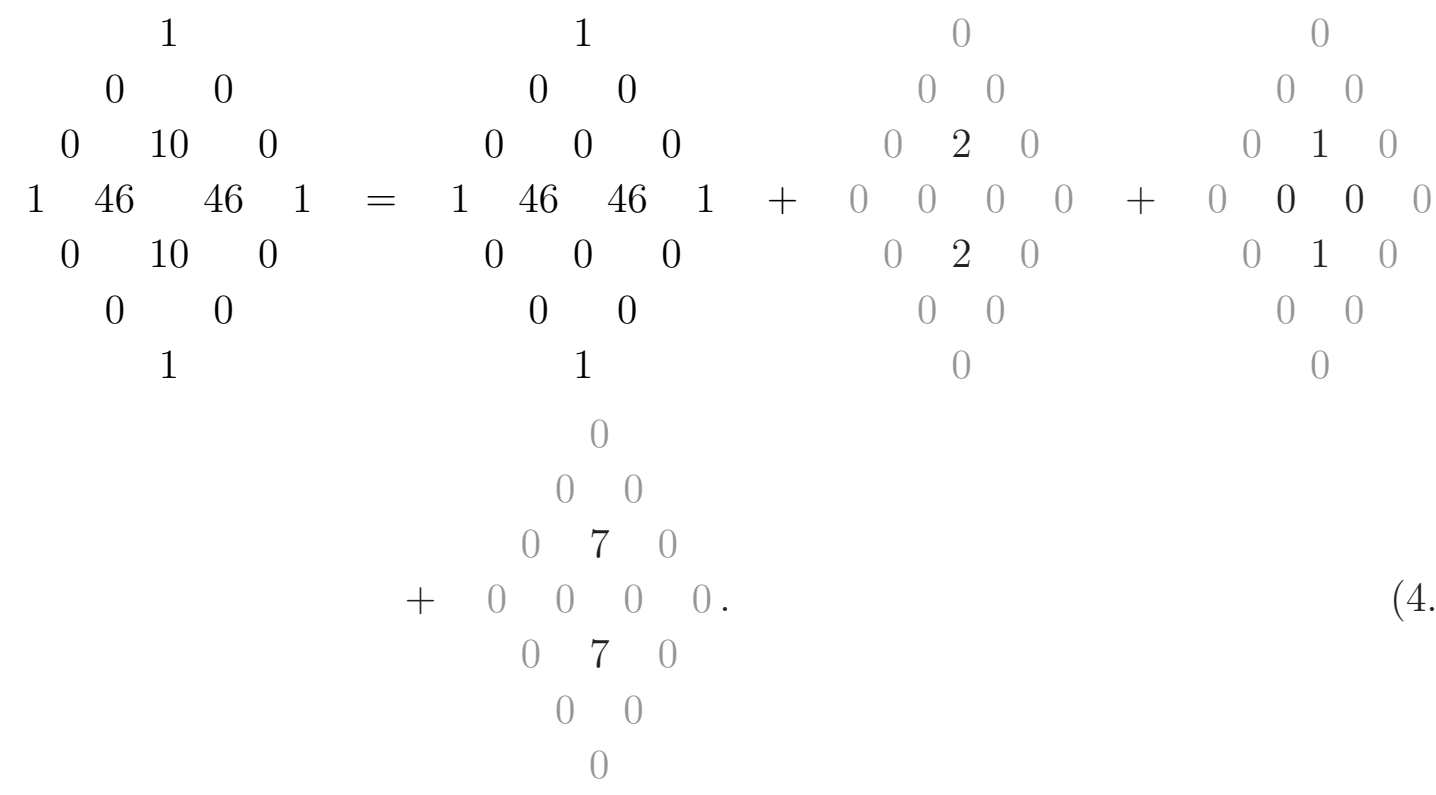

The first three terms on the right-hand side of (4.4) have the same origin as the corresponding terms in Example 4.9. The fourth term includes the contribution of 
the fixed point set associated with the fourth twisted sector (the lower 7) and the fixed point set associated with the ninth twisted sector (the upper 7). Our result (4.4) agrees with the Hodge diamond of the associated Landau-Ginzburg orbifold. For all other fermionic weight assigments consistent with (1.9), we do not obtain this agreement. Thus, in this example, using these heuristic arguments, we obtain a unique result

$$
\left(n_{\eta_{1}}, n_{\eta_{2}}\right)=(3,9) .
$$

In contrast, Conjecture 1.1 allows

$$
\left(n_{\eta_{1}}, n_{\eta_{2}}\right) \in\{(3,9),(6,6)\}
$$

and, as indicated by hypersurface family 8 of the Appendix, the singular locus constraint (1.8) allows

$$
\left(n_{\eta_{1}}, n_{\eta_{2}}\right) \in\{(1,11),(2,10),(3,9),(4,8),(5,7),(6,6)\} .
$$

Example 4.4 A hypersurface in the family $\mathbf{W S P}\left(2,3,1,2,2,2,4 \mid n_{\eta_{1}}, n_{\eta_{2}}\right)[8]$ can be obtained from a Gepner model with level/invariant structure

$$
6_{D} 6_{A} 2_{A} 2_{A} 2_{A}
$$

Following the procedure described in Section 2 , we obtain the modified superpotential

$$
\widetilde{W}=x_{1}^{4}+x_{1} y_{1}^{2}+x_{2}^{8}+x_{3}^{4}+x_{4}^{4}+x_{5}^{4}+z^{2}+\eta_{1} \eta_{2} .
$$

Employing the heuristic reasoning of [4], we find for $\left(n_{\eta_{1}}, n_{\eta_{2}}\right)=(4,4)$ that the Hodge diamond of $\mathcal{M}$ is

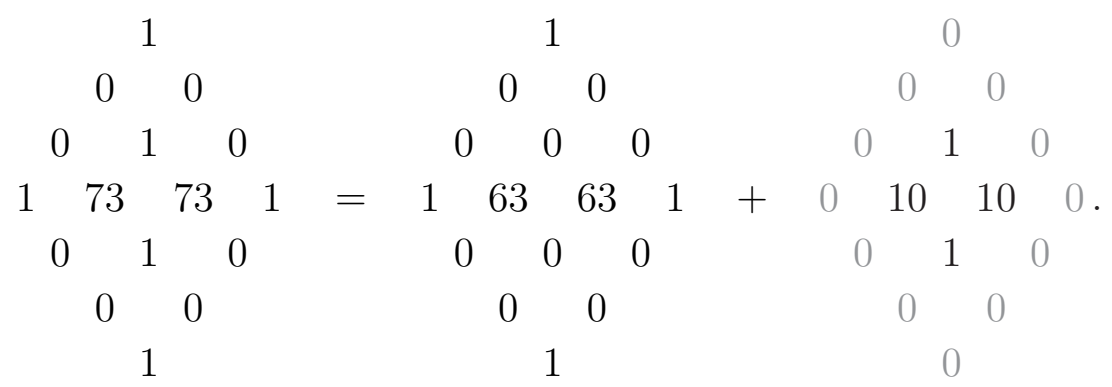

The first term on the right-hand side of (4.5) is the contribution arising from the untwisted sector whereas the second term arises from the fourth twisted sector. Our result (4.5) agrees with the Hodge diamond of the associated Landau-Ginzburg orbifold. For all other fermionic weight assigments consistent with (1.9), we do not obtain this agreement. Thus, in this example, using these heuristic arguments, we obtain a unique result

$$
\left(n_{\eta_{1}}, n_{\eta_{2}}\right)=(4,4) .
$$

In contrast, Conjecture 1.1 and, as indicated by hypersurface family 3 of the Appendix, the singular locus constraint (1.8) both allow

$$
\left(n_{\eta_{1}}, n_{\eta_{2}}\right) \in\{(2,6),(4,4)\} .
$$


Example 4.5 Let us revisit the family of hypersurfaces discussed in Example 3.1, $\operatorname{WSP}\left(2,5,2,5,4,4,4,4,6 \mid n_{\eta_{1}}, n_{\eta_{2}}, n_{\eta_{3}}, n_{\eta_{4}}\right)[12]$. We again focus on the hypersurface

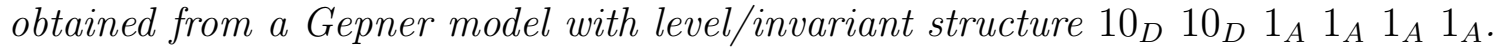
Proceeding as in the above examples, we find for $\left(n_{\eta_{1}}, n_{\eta_{2}}, n_{\eta_{3}}, n_{\eta_{4}}\right)=(2,10,4,8)$ that the Hodge diamond of $\mathcal{M}$ is

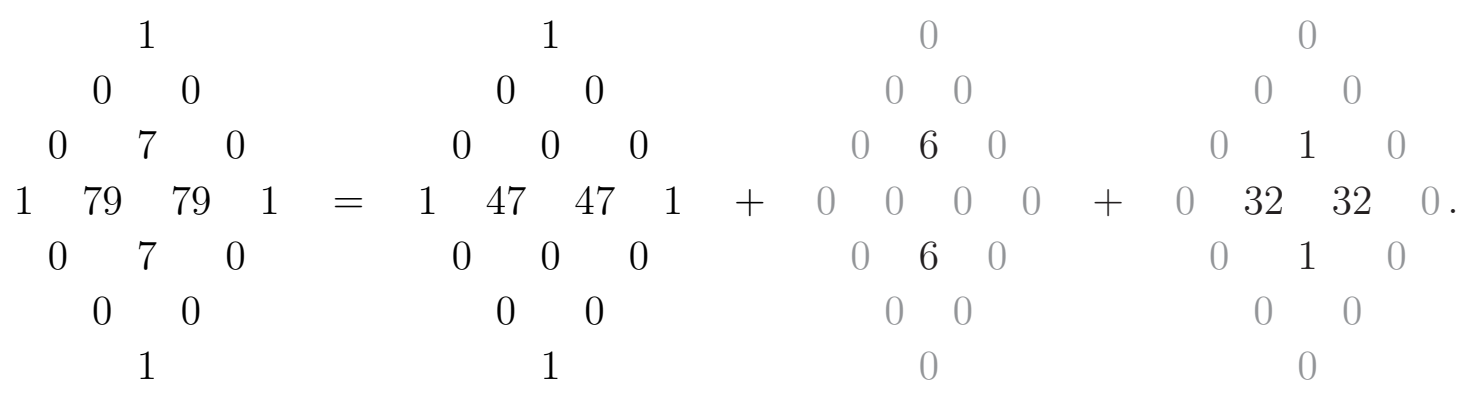

The terms on the right-hand side of (4.9) originate from the same sectors as the corresponding terms in Example 4.9 . Our result (4.0) agrees with the Hodge diamond of the associated Landau-Ginzburg orbifold. For all other fermionic weight assigments consistent with (1.9), we do not obtain this agreement. Thus, in this example, using these heuristic arguments, we obtain

$$
\left(n_{\eta_{1}}, n_{\eta_{2}}, n_{\eta_{3}}, n_{\eta_{4}}\right) \in\{(2,10,4,8),(4,8,6,6)\} .
$$

In contrast, Conjecture 1.1 allows

$$
\left(n_{\eta_{1}}, n_{\eta_{2}}, n_{\eta_{3}}, n_{\eta_{4}}\right) \in\{(2,10,4,8),(4,8,4,8),(4,8,6,6)\}
$$

and the singular locus constraint (1.8) allows

$$
\begin{aligned}
\left(n_{\eta_{1}}, n_{\eta_{2}}, n_{\eta_{3}}, n_{\eta_{4}}\right) \in\{ & (2,10,2,10),(2,10,4,8),(2,10,6,6), \\
& (4,8,4,8),(4,8,6,6),(6,6,6,6)\} .
\end{aligned}
$$

It is interesting to note that, in the above examples, the solutions obtained from the heuristic approach agree precisely with those obtained from Conjecture 1.1 supplemented by the constraint

$$
\widetilde{\mathcal{D}}_{p} \geq 0 \quad \text { whenever } \quad \mathcal{D}_{p} \geq 0 .
$$

Let us now consider an example in which the heuristic approach yields no solution.

Example 4.6 A hypersurface in the family $\mathbf{W S P}\left(1,1,1,2,2,2,3 \mid n_{\eta_{1}}, n_{\eta_{2}}\right)[6]$ can be obtained from a Gepner model with either of the following level/invariant structures:

$$
4_{D} 4_{A} 4_{A} 4_{A} 1_{A}, \quad 4_{A} 4_{A} 4_{A} 1_{A} 1_{A} 1_{A} .
$$


For definiteness, we will focus on the last of these. Following the procedure described in Section \&, we obtain the modified superpotential

$$
\widetilde{W}=x_{1}^{6}+x_{2}^{6}+x_{3}^{6}+x_{4}^{3}+x_{5}^{3}+x_{6}^{3}+z^{2}+\eta_{1} \eta_{2} \text {. }
$$

Employing the heuristic reasoning of [4]/, we find for $\left(n_{\eta_{1}}, n_{\eta_{2}}\right) \in\{(1,5),(3,3)\}$ that the Hodge diamond of $\mathcal{M}$ is

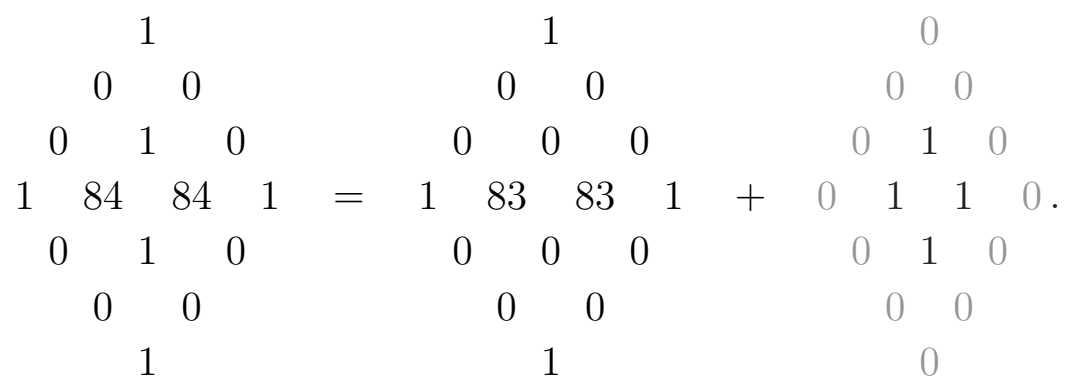

The first term on the right-hand side of (4.8) is the contribution arising from the untwisted sector. The second term includes the contribution of the fixed point set associated with the third twisted sector. For $\left(n_{\eta_{1}}, n_{\eta_{2}}\right)=(2,4)$, we find that the only contribution to the Hodge diamond of $\mathcal{M}$ arises from the untwisted sector. In all cases, the result disagrees with the Landau-Ginzburg orbifold Hodge diamond

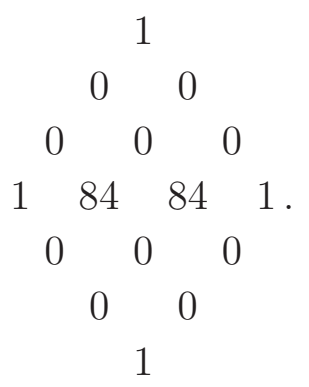

Thus, in this example, using these heuristic arguments, we find no solution for the fermionic weights. In contrast, Conjecture 1.1 and, as indicated by hypersurface family 2 of the Appendix, the singular locus constraint (1.8) both allow $\left(n_{\eta_{1}}, n_{\eta_{2}}\right) \in$ $\{(1,5),(2,4),(3,3)\}$.

\section{Conclusion}

The analysis in Section 4 compares the fermionic weights obtained from our computer program with those obtained by requiring agreement between the Hodge diamond of the Landau-Ginzburg orbifold and the heuristically determined Hodge diamond of the supervariety. Running the program with the singular locus constraint (1.8) yields at least one solution for each $\hat{c}=3$ Gepner model associated with a supervariety through Sethi's proposed correspondence. Conjecture 1.1 is a stronger constraint, 
but it still yields at least one solution for the vast majority of these models. The heuristic approach places the strongest constraint on the fermionic weights. It yields a unique solution in Examples 4.1, 4.2, 4.3, and 4.4, two solutions in Example 4.5, and no solution in Example 4.6. In the examples we have studied, the heuristically determined solutions are a subset of the solutions obtained from Conjecture 1.1. Furthermore, in these examples, when the heuristic approach yields solutions, these solutions agree precisely with those obtained from Conjecture 1.1 supplemented by the constraint (4.7). Thus, something seems to be "right" about the combination of Conjecture 1.1 and the constraint (4.7). A proper supercohomology theory would allow more conclusive statements to be made.

In the Appendix, Table 2 indicates when the Newton polytope of $\widetilde{W}_{\text {bos }}$ admits a nef partition. In this case, the Landau-Ginzburg orbifold can be given a geometrical interpretation as a nonlinear sigma model on a complete intersection Calabi-Yau manifold. The complete intersection Calabi-Yau manifold should be equivalent to the Calabi-Yau supermanifold prescribed by Sethi's proposal. It can be shown that a reflexive Gorenstein polytope $\Delta$ admits a nef partition if and only if the reflexive Gorenstein cones $\sigma_{\Delta}$ and $\sigma_{\Delta}^{\vee}$ are both completely split [8]. In fact, the LandauGinzburg orbifold can be given a complete intersection Calabi-Yau manifold interpretation even when only $\sigma_{\Delta}$ is completely split [8]. In Example 4.4, the Newton polytope of $\widetilde{W}_{b o s}$ is nonreflexive Gorenstein. It turns out that, for all of the remaining examples in Section 4 , the Newton polytope of $\widetilde{W}_{\text {bos }}$ is reflexive Gorenstein but $\sigma_{\Delta}$ is not completely split. Thus, in these examples, the Landau-Ginzburg orbifold cannot be given a complete intersection Calabi-Yau manifold interpretation. We leave a detailed investigation of the cases in which only $\sigma_{\Delta}$ or only $\sigma_{\Delta}^{\vee}$ is completely split to future work.

\section{A. Supervariety hypersurface families}

In Table 2, we list the supervariety hypersurface families associated with $\hat{c}=3$ Gepner models. A hypersurface family corresponding to a hypersurface $\widetilde{W}=0$ which defines a supervariety $\mathcal{M}$ embedded in $\operatorname{WSP}\left(n_{z_{1}}, \ldots, n_{z_{n+1}} \mid n_{\eta_{1}}, \ldots, n_{\eta_{2 m}}\right)$ is denoted by $\mathbf{W S P}\left(n_{z_{1}}, \ldots, n_{z_{n+1}} \mid n_{\eta_{1}}, \ldots, n_{\eta_{2 m}}\right)[d]$. Here, $\widetilde{W}$ is the modified superpotential obtained by satisfying (2.8) and $d$ is its degree of quasihomogeneity. The fermionic weights are determined by requiring (11.8) and (1.9) to be satisfied. The solutions for these fermionic weights are parameterized by $k=1, \ldots,\left[\frac{d}{2 s_{k}}\right]$ and $l=1, \ldots,\left[\frac{d}{2 s_{l}}\right]$, where $s_{k}$ and $s_{l}$ are the coefficients of $k$ and $l$ in the first and third fermion weight assignments, respectively. For each hypersurface family, the Hodge numbers $h^{1,1}$ and $h^{2,1}$ and the Euler number $\chi=2\left(h^{1,1}-h^{2,1}\right)$ of the associated Landau-Ginzburg

orbifold are given. When the Newton polytope of $\widetilde{W}_{\text {bos }}$ admits a nef partition, this is indicated by "nef". In a number of cases, the Landau-Ginzburg orbifold can be given a geometrical interpretation as a product of a two-torus and a K3 surface, which is 
indicated by " $T^{2} \times K 3$ ". Finally, when the Newton polytope of $\widetilde{W}_{\text {bos }}$ is nonreflexive Gorenstein, this is indicated by "nonRG".

Table 2: Supervariety hypersurface families associated with $\hat{c}=3$ Gepner models.

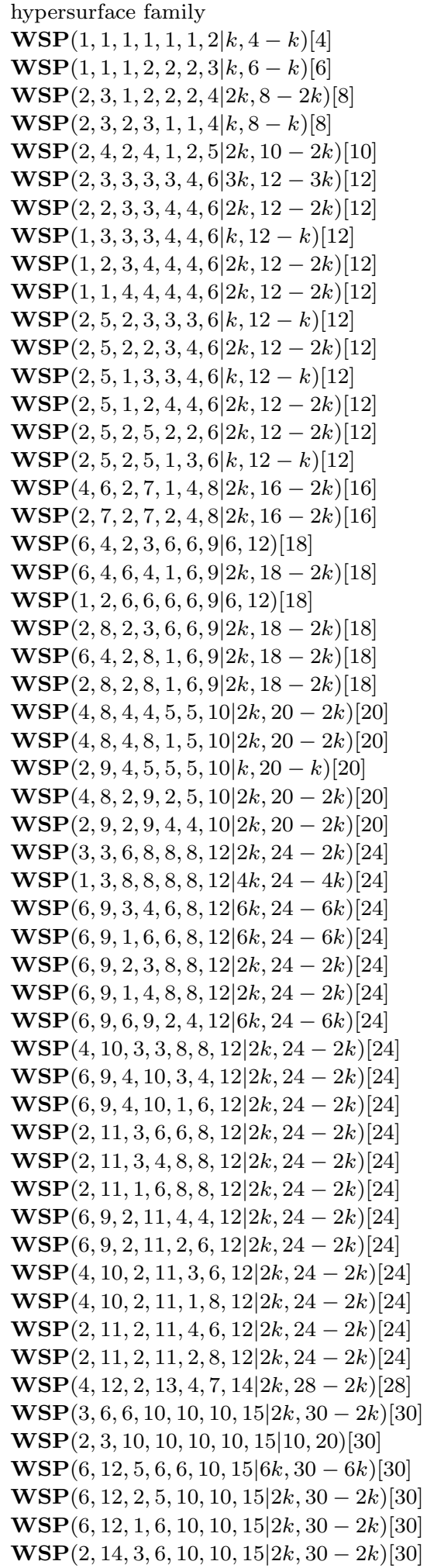

\begin{tabular}{|c|c|c|c|}
\hline$h^{1,1}$ & $h^{2,1}$ & $\chi$ & Comments \\
\hline 0 & 90 & -180 & \\
\hline 0 & 84 & -168 & \\
\hline 1 & 73 & -144 & nonRG \\
\hline 1 & 77 & -152 & \\
\hline 1 & 85 & -168 & \\
\hline 1 & 61 & -120 & \\
\hline 3 & 51 & -96 & \\
\hline 10 & 46 & -72 & \\
\hline 2 & 62 & -120 & \\
\hline 7 & 79 & -144 & \\
\hline 9 & 39 & -60 & \\
\hline 2 & 74 & -144 & nonRG \\
\hline 1 & 61 & -120 & nonRG \\
\hline 3 & 75 & -144 & nonRG \\
\hline 2 & 128 & -252 & \\
\hline 3 & 69 & -132 & \\
\hline 3 & 75 & -144 & \\
\hline 4 & 148 & -288 & nef \\
\hline 2 & 62 & -120 & nonRG \\
\hline 2 & 56 & -108 & \\
\hline 8 & 68 & -120 & \\
\hline 8 & 68 & -120 & \\
\hline 4 & 76 & -144 & \\
\hline 2 & 110 & -216 & \\
\hline 21 & 21 & 0 & nef, $T^{2} \times K 3$ \\
\hline 13 & 49 & -72 & \\
\hline 17 & 29 & -24 & \\
\hline 7 & 79 & -144 & nonRG \\
\hline 7 & 143 & -272 & nef \\
\hline 21 & 21 & 0 & nef, $T^{2} \times K 3$ \\
\hline 16 & 52 & -72 & \\
\hline 3 & 51 & -96 & \\
\hline 10 & 46 & -72 & nonRG \\
\hline 13 & 37 & -48 & nonRG \\
\hline 12 & 48 & -72 & nonRG \\
\hline 6 & 66 & -120 & nef \\
\hline 11 & 35 & -48 & \\
\hline 11 & 35 & -48 & \\
\hline 7 & 55 & -96 & nonRG \\
\hline 10 & 46 & -72 & \\
\hline 12 & 48 & -72 & nonRG \\
\hline 9 & 81 & -144 & \\
\hline 6 & 90 & -168 & nef \\
\hline 6 & 114 & -216 & nef \\
\hline 7 & 55 & -96 & nonRG \\
\hline 3 & 99 & -192 & \\
\hline 8 & 164 & -312 & nef \\
\hline 3 & 243 & -480 & nef \\
\hline 8 & 80 & -144 & \\
\hline 21 & 21 & 0 & nef, $T^{2} \times K 3$ \\
\hline 17 & 41 & -48 & \\
\hline 21 & 21 & 0 & nef, $T^{2} \times K 3$ \\
\hline 17 & 41 & -48 & \\
\hline 7 & 55 & -96 & nonRG \\
\hline 7 & 55 & -96 & \\
\hline
\end{tabular}




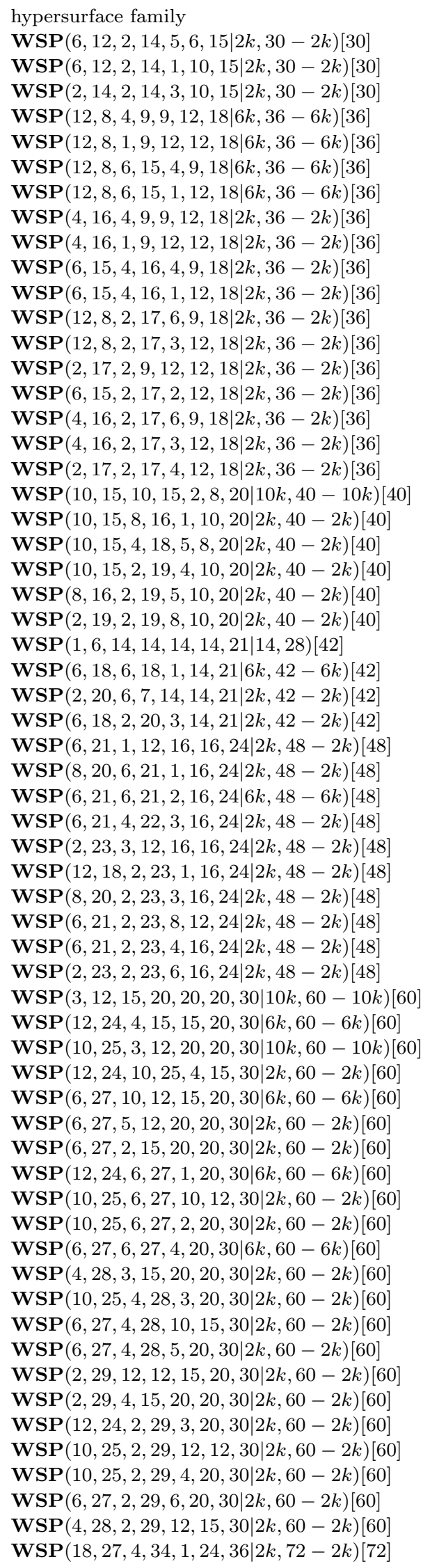

\begin{tabular}{|c|c|c|c|}
\hline$h^{1,1}$ & $h^{2,1}$ & $\chi$ & Comments \\
\hline 7 & 55 & -96 & nonRG \\
\hline 5 & 101 & -192 & \\
\hline 5 & 101 & -192 & \\
\hline 21 & 21 & 0 & nef, $T^{2} \times K 3$ \\
\hline 10 & 46 & -72 & \\
\hline 8 & 44 & -72 & nonRG \\
\hline 13 & 49 & -72 & nonRG \\
\hline 21 & 21 & 0 & nef, $T^{2} \times K 3$ \\
\hline 20 & 56 & -72 & \\
\hline 14 & 50 & -72 & \\
\hline 5 & 77 & -144 & \\
\hline 11 & 53 & -84 & \\
\hline 13 & 49 & -72 & nonRG \\
\hline 16 & 100 & -168 & \\
\hline 5 & 185 & -360 & nef \\
\hline 13 & 73 & -120 & nonRG \\
\hline 5 & 77 & -144 & nonRG \\
\hline 7 & 271 & -528 & nef \\
\hline 7 & 63 & -112 & nef \\
\hline 13 & 49 & -72 & \\
\hline 19 & 27 & -16 & \\
\hline 12 & 96 & -168 & nef \\
\hline 13 & 49 & -72 & \\
\hline 11 & 227 & -432 & nef \\
\hline 23 & 47 & -48 & \\
\hline 15 & 63 & -96 & \\
\hline 23 & 47 & -48 & \\
\hline 15 & 63 & -96 & \\
\hline 20 & 56 & -72 & \\
\hline 8 & 68 & -120 & \\
\hline 7 & 127 & -240 & nef \\
\hline 17 & 41 & -48 & \\
\hline 20 & 56 & -72 & \\
\hline 9 & 129 & -240 & \\
\hline 8 & 68 & -120 & nonRG \\
\hline 16 & 112 & -192 & \\
\hline 8 & 164 & -312 & nonRG \\
\hline 9 & 321 & -624 & nef \\
\hline 21 & 21 & 0 & nef, $T^{2} \times K 3$ \\
\hline 21 & 21 & 0 & nef, $T^{2} \times K 3$ \\
\hline 23 & 23 & 0 & \\
\hline 29 & 29 & 0 & \\
\hline 15 & 39 & -48 & \\
\hline 23 & 23 & 0 & \\
\hline 31 & 55 & -48 & \\
\hline 17 & 65 & -96 & \\
\hline 13 & 49 & -72 & \\
\hline 10 & 106 & -192 & nonRG \\
\hline 11 & 107 & -192 & nef \\
\hline 31 & 31 & 0 & \\
\hline 10 & 46 & -72 & nonRG \\
\hline 25 & 37 & -24 & nonRG \\
\hline 10 & 46 & -72 & nonRG \\
\hline 23 & 47 & -48 & \\
\hline 26 & 86 & -120 & \\
\hline 17 & 65 & -96 & \\
\hline 25 & 85 & -120 & \\
\hline 11 & 155 & -188 & nef \\
\hline 10 & 178 & -336 & \\
\hline 17 & 101 & -168 & \\
\hline 14 & 98 & -168 & \\
\hline
\end{tabular}




\begin{tabular}{|c|c|c|c|c|c|}
\hline$\#$ & hypersurface family & $h^{1,1}$ & $h^{2,1}$ & $\chi$ & Comments \\
\hline 117 & $\mathbf{W S P}(18,27,2,35,8,18,36 \mid 2 k, 72-2 k)[72]$ & 19 & 91 & -144 & nef \\
\hline 118 & $\mathbf{W S P}(18,27,2,35,2,24,36 \mid 2 k, 72-2 k)[72]$ & 14 & 242 & -456 & \\
\hline 119 & $\mathbf{W S P}(6,33,2,35,8,24,36 \mid 2 k, 72-2 k)[72]$ & 15 & 183 & -336 & \\
\hline 120 & $\mathbf{W S P}(4,34,2,35,9,24,36 \mid 2 k, 72-2 k)[72]$ & 14 & 98 & -168 & nonRG \\
\hline 121 & $\mathbf{W S P}(12,36,1,21,28,28,42 \mid 2 k, 84-2 k)[84]$ & 45 & 45 & 0 & \\
\hline 122 & $\mathbf{W S P}(14,35,12,36,1,28,42 \mid 2 k, 84-2 k)[84]$ & 15 & 63 & -96 & \\
\hline 123 & $\mathbf{W S P}(6,39,4,21,28,28,42 \mid 2 k, 84-2 k)[84]$ & 41 & 41 & 0 & \\
\hline 124 & $\mathbf{W S P}(14,35,6,39,4,28,42 \mid 2 k, 84-2 k)[84]$ & 16 & 76 & -120 & nonRG \\
\hline 125 & $\mathbf{W S P}(4,40,12,21,21,28,42 \mid 2 k, 84-2 k)[84]$ & 21 & 21 & 0 & nef, $T^{2} \times K 3$ \\
\hline 126 & $\mathbf{W S P}(14,35,4,40,12,21,42 \mid 2 k, 84-2 k)[84]$ & 35 & 35 & 0 & \\
\hline 127 & $\mathbf{W S P}(2,41,6,21,28,28,42 \mid 2 k, 84-2 k)[84]$ & 40 & 76 & -72 & \\
\hline 128 & $\mathbf{W S P}(14,35,2,41,6,28,42 \mid 2 k, 84-2 k)[84]$ & 15 & 147 & -264 & nef \\
\hline 129 & $\mathbf{W S P}(12,36,2,41,14,21,42 \mid 2 k, 84-2 k)[84]$ & 34 & 58 & -48 & \\
\hline 130 & $\mathbf{W S P}(12,36,2,41,7,28,42 \mid 2 k, 84-2 k)[84]$ & 15 & 63 & -96 & \\
\hline 131 & $\mathbf{W S P}(2,41,2,41,12,28,42 \mid 2 k, 84-2 k)[84]$ & 11 & 491 & -960 & nef \\
\hline 132 & $\mathbf{W S P}(30,20,18,36,1,30,45 \mid 6 k, 90-6 k)[90]$ & 29 & 41 & -24 & nonRG \\
\hline 133 & $\mathbf{W S P}(18,36,10,40,1,30,45 \mid 2 k, 90-2 k)[90]$ & 17 & 65 & -96 & \\
\hline 134 & $\mathbf{W S P}(30,20,2,44,9,30,45 \mid 2 k, 90-2 k)[90]$ & 29 & 41 & -24 & nonRG \\
\hline 135 & $\mathbf{W S P}(18,36,2,44,5,30,45 \mid 2 k, 90-2 k)[90]$ & 17 & 65 & -96 & \\
\hline 136 & $\mathbf{W S P}(10,40,2,44,9,30,45 \mid 2 k, 90-2 k)[90]$ & 17 & 65 & -96 & \\
\hline 137 & $\mathbf{W S P}(24,36,6,45,1,32,48 \mid 6 k, 96-6 k)[96]$ & 24 & 84 & -120 & \\
\hline 138 & $\mathbf{W S P}(24,36,2,47,3,32,48 \mid 2 k, 96-2 k)[96]$ & 24 & 84 & -120 & \\
\hline 139 & $\mathbf{W S P}(6,45,2,47,12,32,48 \mid 2 k, 96-2 k)[96]$ & 18 & 222 & -408 & \\
\hline 140 & $\mathbf{W S P}(30,45,1,24,40,40,60 \mid 10 k, 120-10 k)[120]$ & 39 & 39 & 0 & nonRG \\
\hline 141 & $\mathbf{W S P}(24,48,10,55,3,40,60 \mid 2 k, 120-2 k)[120]$ & 29 & 29 & 0 & \\
\hline 142 & $\mathbf{W S P}(30,45,8,56,1,40,60 \mid 2 k, 120-2 k)[120]$ & 24 & 84 & -120 & \\
\hline 143 & $\mathbf{W S P}(30,45,6,57,2,40,60 \mid 6 k, 120-6 k)[120]$ & 23 & 143 & -240 & \\
\hline 144 & $\mathbf{W S P}(24,48,6,57,5,40,60 \mid 6 k, 120-6 k)[120]$ & 29 & 29 & 0 & \\
\hline 145 & $\mathbf{W S P}(10,55,6,57,12,40,60 \mid 2 k, 120-2 k)[120]$ & 22 & 82 & -120 & \\
\hline 146 & $\mathbf{W S P}(30,45,4,58,3,40,60 \mid 2 k, 120-2 k)[120]$ & 29 & 53 & -48 & \\
\hline 147 & $\mathbf{W S P}(6,57,4,58,15,40,60 \mid 2 k, 120-2 k)[120]$ & 29 & 53 & -48 & nonRG \\
\hline 148 & $\mathbf{W S P}(2,59,15,24,40,40,60 \mid 2 k, 120-2 k)[120]$ & 39 & 39 & 0 & \\
\hline 149 & $\mathbf{W S P}(30,45,2,59,20,24,60 \mid 2 k, 120-2 k)[120]$ & 33 & 69 & -72 & \\
\hline 150 & $\mathbf{W S P}(30,45,2,59,4,40,60 \mid 2 k, 120-2 k)[120]$ & 24 & 204 & -360 & \\
\hline 151 & $\mathbf{W S P}(10,55,2,59,24,30,60 \mid 2 k, 120-2 k)[120]$ & 33 & 141 & -216 & \\
\hline 152 & $\mathbf{W S P}(8,56,2,59,15,40,60 \mid 2 k, 120-2 k)[120]$ & 24 & 84 & -120 & \\
\hline 153 & $\mathbf{W S P}(10,65,4,68,28,35,70 \mid 2 k, 140-2 k)[140]$ & 47 & 47 & 0 & \\
\hline 154 & $\mathbf{W S P}(28,56,2,69,20,35,70 \mid 2 k, 140-2 k)[140]$ & 53 & 53 & 0 & \\
\hline 155 & $\mathbf{W S P}(2,77,12,39,52,52,78 \mid 2 k, 156-2 k)[156]$ & 71 & 71 & 0 & \\
\hline 156 & $\mathbf{W S P}(26,65,2,77,12,52,78 \mid 2 k, 156-2 k)[156]$ & 23 & 143 & -240 & nef \\
\hline 157 & $\mathbf{W S P}(42,63,12,78,1,56,84 \mid 6 k, 168-6 k)[168]$ & 38 & 74 & -72 & \\
\hline 158 & $\mathbf{W S P}(42,63,8,80,3,56,84 \mid 2 k, 168-2 k)[168]$ & 39 & 39 & 0 & \\
\hline 159 & $\mathbf{W S P}(42,63,6,81,4,56,84 \mid 6 k, 168-6 k)[168]$ & 33 & 105 & -144 & \\
\hline 160 & $\mathbf{W S P}(8,80,6,81,21,56,84 \mid 2 k, 168-2 k)[168]$ & 39 & 39 & 0 & \\
\hline 161 & $\mathbf{W S P}(42,63,2,83,6,56,84 \mid 2 k, 168-2 k)[168]$ & 34 & 190 & -312 & \\
\hline 162 & $\mathbf{W S P}(12,78,2,83,21,56,84 \mid 2 k, 168-2 k)[168]$ & 38 & 74 & -72 & \\
\hline 163 & $\mathbf{W S P}(6,81,2,83,24,56,84 \mid 2 k, 168-2 k)[168]$ & 23 & 335 & -624 & \\
\hline 164 & $\mathbf{W S P}(18,81,2,89,20,60,90 \mid 2 k, 180-2 k)[180]$ & 42 & 150 & -216 & \\
\hline 165 & $\mathbf{W S P}(54,81,2,107,8,72,108 \mid 2 k, 216-2 k)[216]$ & 48 & 180 & -264 & \\
\hline 166 & $\mathbf{W S P}(20,100,2,109,44,55,110 \mid 2 k, 220-2 k)[220]$ & 71 & 71 & 0 & \\
\hline 167 & $\mathbf{W S P}(48,96,30,105,1,80,120 \mid 6 k, 240-6 k)[240]$ & 53 & 53 & 0 & \\
\hline 168 & $\mathbf{W S P}(48,96,2,119,15,80,120 \mid 2 k, 240-2 k)[240]$ & 53 & 53 & 0 & \\
\hline 169 & $\mathbf{W S P}(30,105,2,119,24,80,120 \mid 2 k, 240-2 k)[240]$ & 50 & 134 & -168 & \\
\hline 170 & $\mathbf{W S P}(66,99,6,129,8,88,132 \mid 6 k, 264-6 k)[264]$ & 57 & 81 & -48 & \\
\hline 171 & $\mathbf{W S P}(78,117,24,144,1,104,156 \mid 6 k, 312-6 k)[312]$ & 69 & 69 & 0 & \\
\hline 172 & $\mathbf{W S P}(78,117,2,155,12,104,156 \mid 2 k, 312-2 k)[312]$ & 66 & 174 & -216 & \\
\hline 173 & $\mathbf{W S P}(24,144,2,155,39,104,156 \mid 2 k, 312-2 k)[312]$ & 69 & 69 & 0 & \\
\hline 174 & $\mathbf{W S P}(14,161,2,167,48,112,168 \mid 2 k, 336-2 k)[336]$ & 47 & 287 & -480 & \\
\hline 175 & $\mathbf{W S P}(14,203,6,207,60,140,210 \mid 2 k, 420-2 k)[420]$ & 59 & 131 & -144 & \\
\hline 176 & $\mathbf{W S P}(150,225,2,299,24,200,300 \mid 2 k, 600-2 k)[600]$ & 119 & 167 & -96 & \\
\hline 177 & $\mathbf{W S P}(42,441,2,461,132,308,462 \mid 2 k, 924-2 k)[924]$ & 137 & 257 & -240 & \\
\hline
\end{tabular}




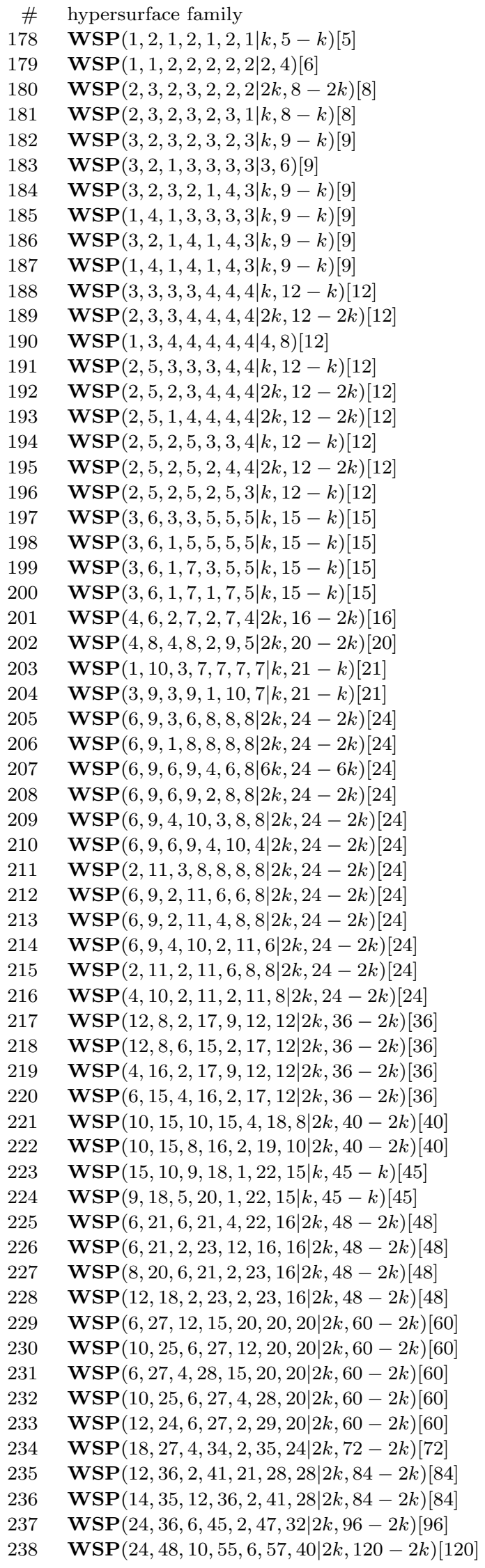

\begin{tabular}{|c|c|c|c|}
\hline$h^{1,1}$ & $h^{2,1}$ & $\chi$ & Comments \\
\hline 1 & 85 & -168 & \\
\hline 1 & 73 & -144 & \\
\hline 2 & 86 & -168 & nef \\
\hline 2 & 58 & -112 & \\
\hline 8 & 35 & -54 & nef \\
\hline 2 & 62 & -120 & nonRG \\
\hline 2 & 56 & -108 & \\
\hline 8 & 68 & -120 & \\
\hline 4 & 76 & -144 & \\
\hline 2 & 110 & -216 & \\
\hline 21 & 21 & 0 & nef, $T^{2} \times K 3$ \\
\hline 11 & 35 & -48 & \\
\hline 2 & 62 & -120 & \\
\hline 4 & 40 & -72 & \\
\hline 2 & 62 & -120 & nonRG \\
\hline 9 & 57 & -96 & \\
\hline 5 & 41 & -72 & \\
\hline 5 & 101 & -192 & nef \\
\hline 3 & 57 & -108 & \\
\hline 21 & 21 & 0 & nef, $T^{2} \times K 3$ \\
\hline 17 & 41 & -48 & \\
\hline 7 & 55 & -96 & nonRG \\
\hline 5 & 101 & -192 & \\
\hline 8 & 104 & -192 & nef \\
\hline 13 & 49 & -72 & \\
\hline 23 & 47 & -48 & \\
\hline 15 & 63 & -96 & \\
\hline 21 & 21 & 0 & nef, $T^{2} \times K 3$ \\
\hline 24 & 36 & -24 & \\
\hline 7 & 55 & -96 & nef \\
\hline 19 & 43 & -48 & nef \\
\hline 9 & 33 & -48 & \\
\hline 20 & 32 & -24 & nef \\
\hline 24 & 36 & -24 & \\
\hline 8 & 68 & -120 & nonRG \\
\hline 10 & 70 & -120 & nonRG \\
\hline 10 & 70 & -120 & nonRG \\
\hline 11 & 131 & -240 & nef \\
\hline 9 & 153 & -288 & nef \\
\hline 10 & 46 & -72 & \\
\hline 17 & 77 & -120 & nonRG \\
\hline 20 & 56 & -72 & \\
\hline 13 & 109 & -192 & nef \\
\hline 31 & 23 & 16 & nef \\
\hline 23 & 59 & -72 & \\
\hline 29 & 41 & -24 & nonRG \\
\hline 17 & 65 & -96 & \\
\hline 31 & 55 & -48 & nef \\
\hline 26 & 86 & -120 & \\
\hline 18 & 102 & -168 & \\
\hline 13 & 229 & -432 & nef \\
\hline 21 & 21 & 0 & nef, $T^{2} \times K 3$ \\
\hline 31 & 31 & 0 & \\
\hline 31 & 31 & 0 & \\
\hline 22 & 58 & -72 & nonRG \\
\hline 21 & 117 & -192 & \\
\hline 22 & 130 & -216 & \\
\hline 45 & 45 & 0 & \\
\hline 37 & 85 & -96 & \\
\hline 26 & 158 & -264 & \\
\hline 49 & 49 & 0 & \\
\hline
\end{tabular}




$\begin{array}{rlrrrr}\# & \text { hypersurface family } & h^{1,1} & h^{2,1} & \chi & \text { Comments } \\ 239 & \mathbf{W S P}(30,45,6,57,4,58,40 \mid 2 k, 120-2 k)[120] & 43 & 67 & -48 & \\ 240 & \mathbf{W S P}(30,45,2,59,24,40,40 \mid 2 k, 120-2 k)[120] & 55 & 55 & 0 & \\ 241 & \mathbf{W S P}(30,45,8,56,2,59,40 \mid 2 k, 120-2 k)[120] & 34 & 118 & -168 & \\ 242 & \mathbf{W S P}(42,63,8,80,6,81,56 \mid 2 k, 168-2 k)[168] & 55 & 55 & 0 & \\ 243 & \mathbf{W S P}(42,63,12,78,2,83,56 \mid 2 k, 168-2 k)[168] & 46 & 106 & -120 & \text { nonRG } \\ 244 & \mathbf{W S P}(48,96,30,105,2,119,80 \mid 2 k, 240-2 k)[240] & 89 & 89 & 0 & \\ 245 & \mathbf{W S P}(78,117,24,144,2,155,104 \mid 2 k, 312-2 k)[312] & 97 & 97 & 0 & \\ 246 & \mathbf{W S P}(1,2,2,2,2,2,2,2,3 \mid 2,4,2,4)[6] & 0 & 84 & -168 & \\ 247 & \mathbf{W S P}(2,3,2,3,2,3,2,3,4 \mid k, 8-k, 2,6)[8] & 1 & 53 & -104 & \\ & \mathbf{W S P}(2,3,2,3,2,3,2,3,4 \mid 3,5,4,4)[8] & 1 & 53 & -104 & \\ 248 & \mathbf{W S P}(3,3,4,4,4,4,4,4,6 \mid 2 k, 12-2 k, 4,8)[12] & 21 & 21 & 0 & T^{2} \times K 3 \\ 249 & \mathbf{W S P}(2,5,3,4,4,4,4,4,6 \mid 2 k, 12-2 k, 4,8)[12] & 2 & 62 & -120 & \text { nonRG } \\ 250 & \mathbf{W S P}(2,5,2,5,4,4,4,4,6 \mid 2 k, 12-2 k, 2 l, 12-2 l)[12] & 7 & 79 & -144 & \\ 251 & \mathbf{W S P}(6,9,6,9,6,8,8,8,12 \mid 2 k, 24-2 k, 6 l, 24-6 l)[24] & 21 & 21 & 0 & \text { nef, } T^{2} \times K 3 \\ 252 & \mathbf{W S P}(6,9,6,9,4,10,8,8,12 \mid 2 k, 24-2 k, 6 l, 24-6 l)[24] & 11 & 35 & -48 & \\ 253 & \mathbf{W S P}(6,9,2,11,8,8,8,8,12 \mid 2 k, 24-2 k, 4 l, 24-4 l)[24] & 16 & 52 & -72 & \\ 254 & \mathbf{W S P}(1,1,1,1,1,1,1,1,1 \mid 1,2,1,2)[3] & 0 & 84 & -168 & \end{array}$

\section{Acknowledgements}

R.G. and M.K. acknowledge financial support from the Austrian Research Funds FWF under grant number P18679-N16.

\section{References}

[1] L. J. Dixon, Some world-sheet properties of superstring compactifications, on orbifolds and otherwise, in Superstrings, unified theories and cosmology 1987, G. Furlan et al., eds., World Scientific 1988;

W. Lerche, C. Vafa and N. P. Warner, Chiral rings in $N=2$ superconformal theories, Nucl. Phys. B 324 (1989) 427;

P. Candelas, M. Lynker and R. Schimmrigk, Calabi-Yau manifolds in weighted $\mathbb{P}_{4}$, Nucl. Phys. B 341 (1990) 383;

B. R. Greene and M. R. Plesser, Duality in Calabi-Yau moduli space, Nucl. Phys. B 338 (1990) 15.

[2] R. Schimmrigk, Critical superstring vacua from noncritical manifolds: A novel framework for string compactifications, Phys. Rev. Lett. 70 (1993) 3688 hep-th/9210062;

R. Schimmrigk, Mirror symmetry and string vacua from a special class of Fano varieties, Int. J. Mod. Phys. A 11 (1996) 3049 hep-th/9405086.

[3] P. Candelas, E. Derrick and L. Parkes, Generalized Calabi-Yau manifolds and the mirror of a rigid manifold, Nucl. Phys. B 407 (1993) 115 hep-th/9304045.

[4] S. Sethi, Supermanifolds, rigid manifolds and mirror symmetry, Nucl. Phys. B 430 (1994) 31 hep-th/9404186].

[5] E. Witten, Phases of $N=2$ theories in two dimensions, Nucl. Phys. B 403 (1993) 159 hep-th/9301042. 
[6] A. Schwarz, Sigma-models having supermanifolds as target spaces, Lett. Math. Phys. 38 (1996) 91 hep-th/9506070.

[7] A. Belhaj, L.B. Drissi, J. Rasmussen, E.H. Saidi and A. Sebbar, Toric Calabi-Yau supermanifolds and mirror symmetry, J. Phys. A 38 (2005) 6405 hep-th/0410291;

S. Seki and K. Sugiyama, Gauged linear sigma model on supermanifold, hep-th/0503074;

R. Ahl Laamara, A. Belhaj, L.B. Drissi, and E.H. Saidi, On local Calabi-Yau supermanifolds and their mirrors, J. Phys. A 39 (2006) 5965 hep-th/0601215.

[8] L. A. Borisov, Towards the mirror symmetry for Calabi-Yau complete intersections in Gorenstein toric Fano varieties, alg-geom/9310001;

V. V. Batyrev and L. A. Borisov, Dual cones and mirror symmetry for generalized Calabi-Yau manifolds, in Mirror Symmetry II, B. Greene and S. T. Yau, eds., Stud. Adv. Math. 1 (1997) 71 alg-geom/9402002;

V. V. Batyrev and B. Nill, Combinatorial aspects of mirror symmetry, Contemp. Math. 452 (2008) 35 math.C0/0703456.

[9] A. Dimca, Singularities and coverings of weighted complete intersections, J. Reine Ang. Math. 366 (1986) 184.

[10] D. Gepner, Exactly solvable string compactifications on manifolds of $S U(N)$ holonomy, Phys. Lett. B 199 (1987) 380;

D. Gepner, Space-time supersymmetry in compactified string theory and superconformal models, Nucl. Phys. B 296 (1988) 757.

[11] C. Vafa, String vacua and orbifoldized L-G models, Mod. Phys. Lett. A 4 (1989) 1169 ;

K. A. Intriligator and C. Vafa, Landau-Ginzburg orbifolds, Nucl. Phys. B 339 (1990) 95.

[12] E. Zaslow, Topological orbifold models and quantum cohomology rings, Commun. Math. Phys. 156 (1993) 301 hep-th/9211119.

[13] C. Vafa and N. P. Warner, Catastrophes and the classification of conformal theories, Phys. Lett. B 218 (1989) 51.

[14] E. J. Martinec, Algebraic geometry and effective Lagrangians, Phys. Lett. B 217 (1989) 431.

[15] S. Cecotti, L. Girardello and A. Pasquinucci, Nonperturbative aspects and exact results for the $N=2$ Landau-Ginzburg models, Nucl. Phys. B 328 (1989) 701;

S. Cecotti, L. Girardello and A. Pasquinucci, Singularity theory and $N=2$ supersymmetry, Int. J. Mod. Phys. A 6 (1991) 2427.

[16] M. Kreuzer and H. Skarke, No mirror symmetry in Landau-Ginzburg spectra!, Nucl. Phys. B 388 (1992) 113 hep-th/9205004. 
[17] P. Di Vecchia, J. L. Petersen and H. B. Zheng, $N=2$ superconformal theory in two dimensions, Phys. Lett. B 162 (1985) 327;

P. Di Vecchia, J. L. Petersen and M. Yu, On the unitary representations of $N=2$ superconformal theory, Phys. Lett. B 172 (1986) 211;

P. Di Vecchia, J. L. Petersen, M. Yu and H. B. Zheng, Explicit construction of unitary representations of the $N=2$ superconformal algebra, Phys. Lett. B 174 (1986) 280;

W. Boucher, D. Friedan and A. Kent, Determinant formulae and unitarity for the $N=2$ superconformal algebra in two dimensions or exact results on string compactification, Phys. Lett. B 172 (1986) 316.

[18] B. R. Greene, C. Vafa and N. P. Warner, Calabi-Yau manifolds and renormalization group flows, Nucl. Phys. B 324 (1989) 371.

[19] R. Schimmrigk, Heterotic $R G$ flow fixed points with non-diagonal affine invariants, Phys. Lett. B 229 (1989) 227;

J. Fuchs, A. Klemm, C. Scheich and M. G. Schmidt, Gepner models with arbitrary affine invariants and the associated Calabi-Yau spaces, Phys. Lett. B 232 (1989) 317;

J. Fuchs, A. Klemm, C. Scheich and M. G. Schmidt, Spectra and symmetries of Gepner models compared to Calabi-Yau compactifications, Ann. Phys. (NY) 204 (1990) 1:

M. Lynker and R. Schimmrigk, A-D-E quantum Calabi-Yau manifolds, Nucl. Phys. B 339 (1990) 121.

[20] M. Kreuzer and H. Skarke, PALP: A Package for Analysing Lattice Polytopes with applications to toric geometry, Comput. Phys. Commun. 157 (2004) 87 math.SC/0204356. 